\title{
Selected Phenotypic Assays Used to Evaluate Antiviral Resistance and Viral Fitness of Hepatitis $B$ Virus and Its Variants
}

\author{
Dieter Glebe Andreas Geipel
}

Institute of Medical Virology, Justus Liebig University Giessen, National Reference Center for Hepatitis B and D

Viruses, German Center for Infection Research (DZIF), Biomedical Research Center Seltersberg, Giessen, Germany

\section{Key Words}

Antiviral resistance $\cdot$ Phenotypic assays · Viral fitness .

Hepatitis B virus

\begin{abstract}
Currently available antiviral therapies specifically target viral replication by blocking reverse transcription with orally given nucleos(t)ide analogues and are able to specifically suppress viral replication. The unique replication strategy of hepatitis B virus (HBV), however, allows long-term persistence of the viral genome within infected hepatocytes in spite of successful therapy. Thus, antiviral therapy needs to be continued for years. Therapy can result either in the emergence and selection of antiviral-resistant variants or the relapse of viral replication after the termination of antiviral therapy. Resistance is a major problem for 4 of the 5 approved HBV nucleos(t)ide analogues, but it is not the only reason for therapy failure. An accurate phenotypic in vitro assay for resistance allows the identification of a viral variant selected in vivo during antiviral therapy and helps to find therapeutic alternatives. Furthermore, these assays can be used to measure viral fitness and pathogenicity in vitro. With the help of these assays, the prediction of emerging viral variants with drug resistance or increased pathogenic potential can be realized. Phenotypic resistance tests for HBV
\end{abstract}

are not trivial because the virus cannot be readily grown in cell culture. This review focuses on currently available phenotypic assays to evaluate antiviral resistance of $\mathrm{HBV}$ and fitness of viral variants in general.

(c) 2014 S. Karger AG, Basel

\section{Introduction}

Replication of hepatitis B virus (HBV) involves a reverse transcription step to convert pregenomic RNA (pgRNA) into relaxed circular viral DNA genome. Reverse transcription during viral genome maturation is prone to inaccuracy due to the missing proofreading function of the enzyme. However, after transmission of wild-type $\mathrm{HBV}$, the genome sequence of the most dominant variant usually appears very stable [1], especially when compared to HIV, which also relies on a reverse transcriptase (RT) for genome replication. Nevertheless, under strong environmental pressure, either by an active immune system or artificially, during antiviral drug therapy, the HBV quasi-species is flexible and able to respond quickly to a given selection pressure. One of the best examples is the fast selection of drug-resistant variants in patients with highly active chronic hepatitis B treated with antivirals of low genetic resistance barrier, like lami-

\section{KARGER}

E-Mail karger@karger.com

www.karger.com/int
(C) 2014 S. Karger AG, Basel

$0300-5526 / 14 / 0574-0225 \$ 39.50 / 0$
Dr. Dieter Glebe

Institute of Medical Virology, Justus Liebig University Giessen, National Reference Center for Hepatitis B and D Viruses, Biomedical Research Center Seltersberg Schubertstrasse 81, DE-35392 Giessen (Germany)

E-Mail dieter.glebe@ viro.med.uni-giessen.de 
vudine [2]. It is the goal of any phenotypic assay to analyze the fitness of selected viral strains under the given selective pressure. In this regard, 'viral fitness' is defined as the ability of a certain viral strain to adapt to a defined environment in order to complete a full replicative cycle, including efficient binding and uptake into its host cells, genome replication, and generation and release of infectious progeny viruses. In vitro assays identify cellular restriction patterns, e.g. the ability to bind efficiently to cellular receptor structures or to provide efficient replication and propagate its viral genome to new host cells. In special animal systems, a phenotypic assay of a viral variant should also be able to evaluate its ability to deal with immunological pressure. However, despite isolation of a high-affinity receptor for HBV [3], no robust and simple system, neither in vitro nor in vivo, exists today that supports propagation of $\mathrm{HBV}$ to analyze the viral fitness of a given strain. Several reliable artificial systems have been evaluated that allow screening of drug resistance or infectivity in vitro and in vivo.

\section{In vitro Phenotypic Assays for HBV Resistance Testing}

The general principle of a phenotypic assay for antiviral resistance is to measure replication of a selected HBV strain containing known or unknown mutations that confer resistance against an antiviral drug interfering with HBV replication. Various assays have been described, all of which have their strengths and weaknesses. The whole approach relies on the detection, isolation, and cloning of HBV DNA from the serum of a chronic hepatitis $B$ patient under antiviral therapy with no or low response to antiviral treatment.

\section{Cell-Free Phenotypic Assays}

Because of the difficulty in synthesis and purification of complete HBV polymerase in the functional form, no robust cell-free assay system for HBV polymerase activity has been established to date. Recently, Tavis et al. [4] succeeded in production of the HBV ribonuclease $\mathrm{H}$ (RNaseH) domain of HBV polymerase in Escherichia coli and its purification via affinity chromatography. Using cloned $\mathrm{RNase} \mathrm{H}$ from HBV genotypes $\mathrm{D}$ and $\mathrm{H}$ in an optimized cell-free system, the authors were able to screen various $\mathrm{RNaseH}$ inhibitors already known to interfere with RNaseH activity of HIV. A couple of active inhibi- tors derived from HIV research have proven to be effective in the cell-free phenotypic assay and were validated in cell culture [4]. Interestingly, RNaseH from HBV genotype $\mathrm{D}$ reacted more sensitively than $\mathrm{RNaseH}$ from genotype $\mathrm{H}$ towards individual inhibitors. Furthermore, Vörös et al. [5] overexpressed and purified two separate parts of HBV polymerase in E. coli: the terminal protein (TP) and a concatemer of RT and RNase H (RT-RH). In an in vitro reconstitution solution, both domains - terminal protein and RT-RH - showed successful interaction resulting in reverse transcription activity that could be blocked by an inhibitor of duck HBV polymerase [5]. Although still not a high-throughput system, these enzymatic assays open a new horizon and offer groundbreaking new tools to screen for novel small-molecule inhibitors interfering with $\mathrm{HBV}$ replication based on the sensitivity of all three domains of the HBV polymerase.

\section{Amplification and Cloning of HBV Genome Variants}

Efficient cloning of drug-resistant HBV genomes from sera of patients is done via PCR amplification by commercially available polymerases. Various polymerases are available that differ in fidelity and error rate. Usually, a reduced error rate caused by proofreading activity of a polymerase results in a lower amplification rate and thus in decreased efficiency of the individual polymerase used. Thus, choosing the right polymerase for amplification is a compromise between the required amount of DNA template (dependent on the viral DNA titer in the sample), the length of the amplificate, time, and number of cycles taken during PCR and fidelity of the used polymerase including its ability for proofreading during the synthesis of the DNA strands. The gold standard of any phenotypic assay using cloned HBV genomes is the amplification and cloning of the full DNA genome $(3.2 \mathrm{~kb})$ via a PCR polymerase with the highest proofreading fidelity available. However, usually $10^{5}$ to $10^{6} \mathrm{HBV}$ genomes per PCR reaction are necessary to obtain a sufficient amount for successful cloning because of the reduced amplification power of those polymerases for long DNAs. Furthermore, the nick and the gap also reduce the amplification efficiency. Consequently, many authors amplify only the RT domain of the HBV polymerase since primary and secondary resistance mutations against nucleos(t)ide analogues have been described to cluster within the RT domain of the HBV polymerase [6]. However, other parts of the polymerase, like the $\mathrm{RNaseH}$ or the primer domain might also have an influence on antiviral 
resistance and thus on fitness of an HBV variant. Cloning of the entire polymerase ORF that accounts for $70 \%$ of the $\mathrm{HBV}$ genome might be a compromise if full genome amplification cannot be achieved. Since the polymerase ORF also contains the full ORF of the HBV surface proteins [for a review, see 7], this approach gives additional detailed genotypic information on further mutations within the HBV surface proteins. For example, many mutations within the first 77 amino acids of the preS1 domain can result in decrease or even loss of infectivity of those mutants [8]. Cloning of the RT domain alone will only give essential information about changes within the $S$ domain, e.g. stop mutations that can lead to secretion-deficient variants [9], or other mutations within the antigenic determinant, responsible for HBV immune escape [10-13] and their transmission [14].

Using an elegant PCR approach, Günther et al. [15] used specially designed PCR primers to amplify fulllength (1.0) genomes from serum of hepatitis B patients. The authors took advantage of the fact that the plus and minus strands of the HBV DNA genome within the virion are not covalently linked [7]. The authors designed primers close to a highly conserved genomic region of $\mathrm{HBV}$ called direct repeat 1 and inserted within their primers an artificial DNA restriction site that allowed the release of a full-genome HBV construct (also called 1.0 $\times$ HBV genome) with self-complementary DNA ends after treatment with respective restriction nucleases [15]. Afterwards, the modified, but still linear full-length HBV DNA double-strand could directly be transfected into hepatoma cell lines (e.g. HuH7), where it is supposed to circularize into cccDNA. The great advantage of this method is a vector-free expression of HBV genomes, derived directly from serum of $\mathrm{HBV}$-infected patients, allowing cloning and expression of all major viral variants from one individual serum. However, viral replication from cccDNA in hepatoma cell lines is usually lower than from HBV overlength expression vectors with strong artificial promoters (e.g. CMV) and thus might be difficult to detect [16].

In contrast to the HBV full-genome PCR method of Günther et al. [15], amplified subgenomic DNA fragments of HBV (e.g. the polymerase ORF or the RT region) have to be inserted into an existing HBV expression vector cassette harboring an HBV genome with overlength orientation. This procedure allows the starting of the replicative cycle of $\mathrm{HBV}$ via transcription of pgRNA from the overlength expression plasmid after transfection into hepatoma cell lines (e.g. HepG2 or HuH7). Based on the layout of the expression plasmid, pgRNA synthesis could either be driven by HBV's own promoters and enhancers

Phenotypic Assays for Evaluating

Antiviral Resistance and Viral Fitness (present either $1.3 \times, 1.5 \times$, or $2.0 \times$ in the genome) or by artificial promoters (usually a strong CMV promoter) for $1.1 \times$ HBV genome constructs [16-18]. If evaluation of HBV pgRNA synthesis is the main goal of the phenotypic assay, e.g. to analyze effects of core and precore promoter mutants, constructs using artificial promoters are not suitable. For those approaches, the method of Günther et al. [15] or the use of HBV 1.5-mers or HBV dimers with HBV natural promoter/enhancer structures should be used.

Since a robust expression of $\mathrm{HBV}$ pgRNA is needed for the investigation of antiviral drug-resistance mutations within the RT domain of the HBV polymerase, constructs with strong external promoters usually provide the most reliable results. However, one has to keep in mind that any insertion of foreign subgenomic fragments into an existing HBV expression plasmid results in the production of chimeric viral particles and the possibility of altered phenotypic characteristics. For example, exchange of the complete polymerase ORF within a $1.1 \times$ overlength HBV expression construct results in synthesis of chimeric core particles and X-protein, due to overlapping ORFs in the HBV genomic architecture. Cloned expression constructs are usually transfected as expression plasmids, but can also be delivered via baculoviral $[19,20]$, adenoviral [21], or adeno-associated virus vectors [22]. Some studies have been done with cell lines stably transfected with a selected HBV expression cassette [23-25]. Generation of stable cell lines is much more time-consuming than transient transfection, but the clonal character of a stable cell line could overcome variability with transient transfection in difficult-to-transfect cell lines, like HepG2. Many of those stable cell lines have been used to determine susceptibility of certain well-characterized drug-resistant $\mathrm{HBV}$ variants to antiviral nucleos(t)ide analogues and to analyze cross-resistance of distinct resistance mutations within the RT domain of the HBV polymerase [23-25]. However, one has to take into account that the integration of the HBV expression cassette into certain loci of the host cell genome is prone to epigenetic variations that could alter the expression level of the HBV clone and thus could bias the phenotypic assay in terms of viral fitness.

\section{Construction of Phenotypic Assays to Analyze Viral Fitness}

Detection of viral DNA replication in transfected cells is done with cytoplasm or the supernatant of the cells. Use of quantitative real-time PCR systems have become wide- 


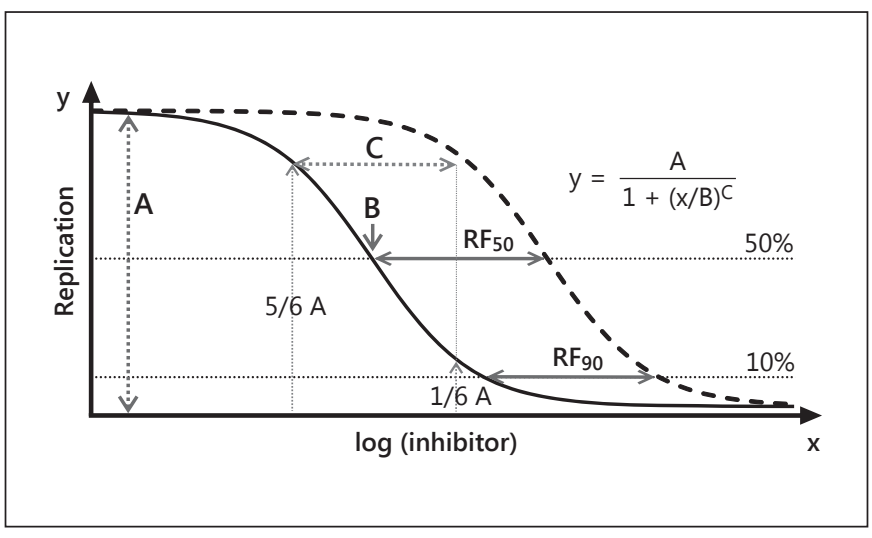

Fig. 1. Example of a dose response function for a viral inhibitor and viral replication using a regression model and calculation of resistance factors. The inhibition curve is described by the equation in the upper right corner, using three different parameters: the amplitude of the curve (difference between no inhibition and maximum inhibition) is defined by parameter $\mathrm{A}$, parameter $\mathrm{B}$ defines the inflexion point (the $\mathrm{x}$ value at $1 / 2 \mathrm{~A}$ ), and parameter $\mathrm{C}$ controls the transition width of the curve (distance on the $\mathrm{x}$-axis between the $x$ values with corresponding $y$ values of $1 / 6 \mathrm{~A}$ and $5 / 6 \mathrm{~A}$ ). The equation is used to calculate the $\mathrm{IC}_{50}$ and the $\mathrm{IC}_{90}$ values. For the quantification of the resistance, the ratio between the wild-type $\mathrm{IC}_{50 / 90}$ and the $\mathrm{IC}_{50 / 90}$ of the isolate is calculated. The resulting value is called the resistance factor $\left(\mathrm{RF}_{50 / 90}\right)$.

ly accepted nowadays in contrast to detection by Southern blot, which is much more tedious and of limited sensitivity and accuracy compared to PCR-based assays [26]. Despite these challenges, Southern blot analysis could provide important additional analytic information about replicative DNA intermediates of a variant and has been very helpful in the characterization of antiviral resistance mutation in the RT rtA181T, resulting in a stop mutation (sW172stop) in the HBV surface proteins [9]. Although PCR assays have the advantage of higher sensitivity of detection of mutations and are thus not fully dependent on robust replication of the HBV variant under investigation, they are more prone to false-positive results, e.g. by contamination with a vector-based DNA template from the viral input. However, PCR assays can be easily downscaled to a 96-well format which is not possible with Southern blot assays.

For quantification of resistance, the half maximal inhibitory concentration $\left(\mathrm{IC}_{50}\right)$ of a wild-type variant is usually compared to the mutant. For the exact definition of the $\mathrm{IC}_{50}$ of a certain mutant, the replication of this variant under certain drug concentrations is measured. The resulting data are then plotted and the sigmoidal inhibition curve is subsequently described via multiparametri- cal nonlinear regression models (fig. 1) [27]. The significance of the differences of replication between the wildtype and the variant can then be tested statistically via univariate ANOVA tests. Due to the multiple comparisons to a similar wild type, a certain procedure should be used to adjust the significance threshold [28], e.g. Bonferroni correction [29].

\section{Phenotypic Assays Based on Infectivity of HBV/HDV: Cell Culture Assays}

Propagation of HBV would be very useful, e.g. in comparing the fitness of two HBV variants or the phenotypic adaptation of different HBV variants under antiviral treatment in vitro. In the past, infectivity assays for $\mathrm{HBV}$ were only possible with primary hepatocytes of proven susceptibility, derived either from humans, higher primates, or the Asian tree shrew Tupaia belangeri $[30,31]$. In 2002, a human hepatoma cell line (HepaRG) was characterized that regained limited susceptibility after cultivation in a special medium requiring high concentration of dimethyl sulfoxide [32]. Since the characterization of human liver bile acid transporter $\mathrm{Na}^{+}$-taurocholate cotransporting polypeptide (hNTCP) as a high-affinity receptor for HBV and HDV [3], human hepatoma cell lines like HepG2 and HuH7 gain HBV susceptibility upon transient or stable expression of cloned hNTCP [3]. For any infectivity assay, HBV and HDV, either from cell culture or patient-derived materials, can be used $[8,33]$. However, a major drawback is the high amount of viral particles that are still needed for optimal infectivity even for cells of hepatoma cell lines, overexpressing hNTCP [3, 33]. Furthermore, production and secretion of infectious HBV from those cultures is far from optimal. These weaknesses might be the reason that all in vitro infection systems to date lack robust propagation and reinfection of the newly produced viruses in culture cells, even after artificial overexpression of NTCP [3]. Nevertheless, with the current infectivity assays, validation of HBV variants is possible if the viral fitness of individual variants allows sufficient production of virions [34].

Production of virions in cell culture from cloned and transfected HBV genomes can be achieved by any of the methods described above. Usually, for improved expression of the virions, HBV expression constructs $(1.1 \times)$ with a strong artificial promoter (e.g. CMV) are currently used. For all in vitro infection assays, standardization of the viral input is crucial. Human plasma from chronic hepatitis B patients usually contains a large surplus of 
subviral particles that could interfere with receptor recognition at the hNTCP. Furthermore, human plasma components have been described to interfere with infection in vitro [35]; therefore, purification of the virions is recommended $[35,36]$. Ultracentrifugation using various density media (sucrose, percoll) will result in enrichment of virions and separation from the bulk of subviral particles and main plasma components [37-39]. Quantification of virions can be achieved by real-time PCR assays using WHO standards [26]. The total amount of HBsAg could be determined with currently available diagnostic systems, or by in-house ELISA systems using specific monoclonal antibodies against all three HBV surface proteins, thus also allowing quantification of large and middle HBV surface proteins [38]. Quantification of viral particles from cell culture is even more challenging since in addition to enveloped virions, those cultures also produce large amounts of nonenveloped DNA-containing core particles [25, 40-42] that are noninfectious [43]. The ratio of viral particles to core particles (HBV) should be determined, e.g. after centrifugation in cesium-chloride density gradients followed by quantification of core and complete HBV particles in the layers [44]. Alternatively, enveloped particles could be quantified by immune precipitation with anti-preS1 antibodies [38]. Purification of virions and separation from core particles can be achieved by ultracentrifugation with density gradients, e.g. sucrose or percoll $[36,37]$, or by affinity purification with heparin columns $[45,46]$. HBV/HDV-infectivity assays have been useful in characterizing neutralizing HBV antibodies [36, 38], validation of antiviral drugs, e.g. nucleos(t)ide analogues [47] and viral entry inhibitors [48, 49], and to analyze the zoonotic potential of new orthohepadnaviruses from bats [33]. To phenotypically analyze potential pathogenic effects of viral variants, primary hepatocytes still have to be used [50].

\section{Phenotypic Assays Using Animal Models}

Due to the high species specificity of HBV, in vivo infectivity studies have been done using chimpanzees ( $P a n$ troglodytes). In an approach to study viral fitness and infectivity of drug-resistant HBV strains, Kamili et al. [51] successfully transmitted a clonal lamivudine-resistant triple HBV variant (rtV173L, rtL180M, and rtM204V) to chimpanzees. This viral variant also showed changes in the overlapping ORF for the surface proteins (sE146D, sI195M), allowing transmission even to successfully vaccinated chimpanzees [51]. Since HBV infection experi-

ments with chimpanzees are difficult to manage and ethically questionable, in vivo infection experiments were also performed with immunodeficient mice harboring a humanized liver, i.e. uPa-Scid mice $[52,53]$. The uPa-Scid mouse system has been successfully used to study the infectivity of HBV and HDV variants [54] and antiviral drug evaluation [55], and to analyze the efficacy of viral entry inhibitors [56, 57]. Furthermore, the direct cytopathic effects of $\mathrm{HBV}$ variants have been evaluated with this model [58]. However, due to the complexity of this animal model and the lack of an adaptive immune system, phenotypic screening of a large array of $\mathrm{HBV}$ variants seems to be difficult. A breakthrough would be the establishment of a small animal model that is transgenic for human NTCP with the ability to fully support the replicative cycle of HBV.

\section{Acknowledgements}

The authors thank Wolfram H. Gerlich, Rolf Kaiser, and Lutz Gürtler for their critical reading of the manuscript. The work of the authors was supported by grants from the German Federal Ministry of Education and Research (BMBF, grant No. 01ES0818 'HOPE-project' to D.G.), the Deutsche Forschungsgemeinschaft (DFG, German Research Foundation), the Germany Federal Ministry of Health, The German Center for Infection Research (DZIF), the Universitätsklinikum Giessen and Marburg $\mathrm{GmbH}$, and the von Behring-Roentgen Foundation, Germany.

\section{Disclosure Statement}

The authors have no conflicts of interest to disclose.

References

Intervirology 2014;57:225-231 DOI: $10.1159 / 000360950$
Phenotypic Assays for Evaluating

Antiviral Resistance and Viral Fitness
- 1 Dreesman JM, Baillot A, Hamschmidt L, Monazahian M, Wend UC, Gerlich WH: Outbreak of hepatitis B in a nursing home associated with capillary blood sampling. Epidemiol Infect 2006;134:1102-1113.

2 Lai CL, Dienstag J, Schiff E, Leung NW, Atkins M, Hunt C, Brown N, Woessner M, Boehme R, Condreay L: Prevalence and clinical correlates of YMDD variants during lamivudine therapy for patients with chronic hepatitis B. Clin Infect Dis 2003;36:687-696.

-3 Yan H, Zhong G, Xu G, He W, Jing Z, Gao Z, Huang Y, Qi Y, Peng B, Wang H, Fu L, Song M, Chen P, Gao W, Ren B, Sun Y, Cai T, Feng $\mathrm{X}$, Sui J, Li W: Sodium taurocholate cotransporting polypeptide is a functional receptor for human hepatitis B and D virus. Elife 2012; 1:e00049. 
-4 Tavis JE, Cheng X, Hu Y, Totten M, Cao F, Michailidis E, Aurora R, Meyers MJ, Jacobsen EJ, Parniak MA, Sarafianos SG: The hepatitis $B$ virus ribonuclease $H$ is sensitive to inhibitors of the human immunodeficiency virus ribonuclease $\mathrm{H}$ and integrase enzymes. PLoS Pathog 2013;9:e1003125.

-5 Vörös J, Urbanek A, Rautureau GJ, O'Connor M, Fisher HC, Ashcroft AE, Ferguson N: Large-scale production and structural and biophysical characterizations of the human hepatitis B virus polymerase. J Virol 2014;88: 2584-2599.

6 Zoulim F, Locarnini S: Hepatitis B virus resistance to nucleos(t)ide analogues. Gastroenterology 2009;137:1593-1608.e1-2.

7 Glebe D, Bremer CM: The molecular virology of hepatitis B virus. Semin Liver Dis 2013;33: 103-112.

8 Glebe D, Urban S: Viral and cellular determinants involved in hepadnaviral entry. World J Gastroenterol 2007;13:22-38.

9 Warner N, Locarnini S: The antiviral drug selected hepatitis B virus rtA181T/sW172* mutant has a dominant negative secretion defect and alters the typical profile of viral rebound. Hepatology 2008;48:88-98.

$\checkmark 10$ Torresi J, Earnest-Silveira L, Deliyannis G, Edgtton K, Zhuang H, Locarnini SA, Fyfe J, Sozzi T, Jackson DC: Reduced antigenicity of the hepatitis B virus HBsAg protein arising as a consequence of sequence changes in the overlapping polymerase gene that are selected by lamivudine therapy. Virology 2002;293: 305-313.

11 Torresi J: The virological and clinical significance of mutations in the overlapping envelope and polymerase genes of hepatitis B virus. J Clin Virol 2002;25:97-106.

12 Sheldon J, Soriano V: Hepatitis B virus escape mutants induced by antiviral therapy. J Antimicrob Chemother 2008;61:766-768.

13 Kwei K, Tang X, Lok AS, Sureau C, Garcia T, Li J, Wands J, Tong S: Impaired virion secretion by hepatitis B virus immune escape mutants and its rescue by wild-type envelope proteins or a second-site mutation. J Virol 2013;87:2352-2357.

14 Thibault V, Aubron-Olivier C, Agut H, Katlama C: Primary infection with a lamivudineresistant hepatitis B virus. AIDS 2002;16:131133.

15 Günther S, Li BC, Miska S, Krüger DH, Meisel $\mathrm{H}$, Will $\mathrm{H}$ : A novel method for efficient amplification of whole hepatitis $B$ virus genomes permits rapid functional analysis and reveals deletion mutants in immunosuppressed patients. J Virol 1995;69:5437-5444.

16 Durantel D, Brunelle M-N, Gros E, CarroueeDurantel S, Pichoud C, Villet S, Trepo C, Zou$\lim$ F: Resistance of human hepatitis B virus to reverse transcriptase inhibitors: from genotypic to phenotypic testing. J Clin Virol 2005; 34(suppl 1):S34-S43.
17 Allen MI, Deslauriers M, Andrews CW, Tipples GA, Walters KA, Tyrrell DL, Brown N, Condreay LD: Identification and characterization of mutations in hepatitis B virus resistant to lamivudine. Lamivudine Clinical Investigation Group. Hepatology 1998;27: 1670-1677.

18 Glebe D, Berting A, Broehl S, Naumann H, Schuster R, Fiedler N, Tolle TK, Nitsche S, Seifer M, Gerlich WH, Schaefer S: Optimised conditions for the production of hepatitis $\mathrm{B}$ virus from cell culture. Intervirology 2001;44: 370-378.

19 Delaney WE 4th, Isom HC: Hepatitis B virus replication in human HepG2 cells mediated by hepatitis $B$ virus recombinant baculovirus. Hepatology 1998;28:1134-1146.

20 Lucifora J, Durantel D, Belloni L, Barraud L, Villet S, Vincent IE, Margeridon-Thermet S, Hantz O, Kay A, Levrero M, Zoulim F: Initiation of hepatitis $\mathrm{B}$ virus genome replication and production of infectious virus following delivery in HepG2 cells by novel recombinant baculovirus vector. J Gen Virol 2008;89: 1819-1828.

21 Ren S, Nassal M: Hepatitis B virus (HBV) virion and covalently closed circular DNA formation in primary tupaia hepatocytes and human hepatoma cell lines upon HBV genome transduction with replication-defective adenovirus vectors. J Virol 2001;75:1104-1116.

22 Dion S, Bourgine M, Godon O, Levillayer F, Michel M-L: Adeno-associated virus-mediated gene transfer leads to persistent hepatitis $\mathrm{B}$ virus replication in mice expressing HLA-A2 and HLA-DR1 molecules. J Virol 2013;87: 5554-5563.

$23 \mathrm{Fu} \mathrm{L}$, Cheng YC: Characterization of novel human hepatoma cell lines with stable hepatitis $B$ virus secretion for evaluating new compounds against lamivudine- and penciclovirresistant virus. Antimicrob Agents Chemother 2000;44:3402-3407.

24 Qi X, Xiong S, Yang H, Miller M, Delaney WE: In vitro susceptibility of adefovir-associated hepatitis $\mathrm{B}$ virus polymerase mutations to other antiviral agents. Antivir Ther 2007; 12:355-362.

25 Sun D, Nassal M: Stable HepG2- and Huh7based human hepatoma cell lines for efficient regulated expression of infectious hepatitis $B$ virus. J Hepatol 2006;45:636-645.

26 Jursch CA, Gerlich WH, Glebe D, Schaefer S, Marie O, Thraenhart O: Molecular approaches to validate disinfectants against human hepatitis B virus. Med Microbiol Immunol 2002;190:189-197.

27 Sampah ME, Shen L, Jilek BL, Siliciano RF: Dose-response curve slope is a missing dimension in the analysis of HIV-1 drug resistance. Proc Natl Acad Sci USA 2011;108: 7613-7618.

28 Curran-Everett D: Multiple comparisons: philosophies and illustrations. Am J Physiol Regul Integr Comp Physiol 2000;279:R1-R8.
29 Bland JM, Altman DG: Multiple significance tests: the Bonferroni method. BMJ 1995;310: 170 .

30 Glebe D: Attachment sites and neutralising epitopes of hepatitis B virus. Minerva Gastroenterol Dietol 2006;52:3-21.

-31 Glebe D: Recent advances in hepatitis B virus research: a German point of view. World J Gastroenterol 2007;13:8-13.

32 Gripon P, Rumin S, Urban S, Le Seyec J, Glaise D, Cannie I, Guyomard C, Lucas J, Trepo C, Guguen-Guillouzo C: Infection of a human hepatoma cell line by hepatitis B virus. Proc Natl Acad Sci USA 2002;99:15655-15660.

33 Drexler JF, Geipel A, König A, et al: Bats carry pathogenic hepadnaviruses antigenically related to hepatitis B virus and capable of infecting human hepatocytes. Proc Natl Acad Sci USA 2013;110:16151-16156.

34 Villet S, Billioud G, Pichoud C, Lucifora J, Hantz O, Sureau C, Deny P, Zoulim F: In vitro characterization of viral fitness of therapy-resistant hepatitis B variants. Gastroenterology 2009;136:168-176.e2.

-35 Köck J, Nassal M, MacNelly S, Baumert TF, Blum HE, von Weizsäcker F: Efficient infection of primary tupaia hepatocytes with purified human and woolly monkey hepatitis B virus. J Virol 2001;75:5084-5089.

36 Glebe D, Aliakbari M, Krass P, Knoop EV, Valerius KP, Gerlich WH: Pre-s1 antigen-dependent infection of Tupaia hepatocyte cultures with human hepatitis B virus. J Virol 2003;77:9511-9521.

-37 Köck J, Glebe D: Hepatitis B virus infection of primary tupaia hepatocytes; in Von Weizsäcker F, Roggendorf M (eds): Models of Viral Hepatitis. Basel, Karger, 2005, vol 25, pp $96-$ 105 .

- 38 Bremer CM, Sominskaya I, Skrastina D, Pumpens P, El Wahed AA, Beutling U, Frank R, Fritz HJ, Hunsmann G, Gerlich WH, Glebe D: N-terminal myristoylation-dependent masking of neutralizing epitopes in the preS1 attachment site of hepatitis B virus. J Hepatol 2011;55:29-37.

39 Glebe D, Gerlich WH: Study of the endocytosis and intracellular localization of subviral particles of hepatitis B virus in primary hepatocytes. Methods Mol Med 2004;96:143-151.

40 Wittkop L, Schwarz A, Cassany A, GrünBernhard S, Delaleau M, Rabe B, Cazenave C, Gerlich W, Glebe D, Kann M: Inhibition of protein kinase $\mathrm{C}$ phosphorylation of hepatitis $B$ virus capsids inhibits virion formation and causes intracellular capsid accumulation. Cell Microbiol 2010;12:962-975.

41 Watanabe T, Sorensen EM, Naito A, Schott M, Kim S, Ahlquist P: Involvement of host cellular multivesicular body functions in hepatitis B virus budding. Proc Natl Acad Sci USA 2007;104:10205-10210.

42 Pairan A, Bruss V: Functional surfaces of the hepatitis B virus capsid. J Virol 2009;83: 11616-11623. 
43 Bremer CM, Bung C, Kott N, Hardt M, Glebe $D$ : Hepatitis $B$ virus infection is dependent on cholesterol in the viral envelope. Cell Microbiol 2009;11:249-260.

-44 Bremer CM, Saniewski M, Wend UC, Torres P, Lelie N, Gerlich WH, Glebe D: Transient occult hepatitis B virus infection in a blood donor with high viremia. Transfusion 2009; 49:1621-1629.

45 Sureau C, Salisse J: A conformational heparan sulfate binding site essential to infectivity overlaps with the conserved hepatitis B virus a-determinant. Hepatology 2013;57:985-994.

46 Schulze A, Gripon P, Urban S: Hepatitis B virus infection initiates with a large surface protein-dependent binding to heparan sulfate proteoglycans. Hepatology 2007;46:17591768.

47 Köck J, Baumert TF, Delaney WEt, Blum HE, von Weizsäcker F: Inhibitory effect of adefovir and lamivudine on the initiation of hepatitis B virus infection in primary tupaia hepatocytes. Hepatology 2003;38:1410-1418.

48 Glebe D, Urban S, Knoop EV, Cag N, Krass P, Grün S, Bulavaite A, Sasnauskas K, Gerlich WH: Mapping of the hepatitis B virus attachment site by use of infection-inhibiting preS1 lipopeptides and tupaia hepatocytes. Gastroenterology 2005;129:234-245.
49 Gripon P, Cannie I, Urban S: Efficient inhibition of hepatitis B virus infection by acylated peptides derived from the large viral surface protein. J Virol 2005;79:1613-1622.

50 Baumert TF, Yang C, Schurmann P, Köck J, Ziegler C, Grullich C, Nassal M, Liang TJ, Blum HE, von Weizsäcker F: Hepatitis B virus mutations associated with fulminant hepatitis induce apoptosis in primary Tupaia hepatocytes. Hepatology 2005;41:247-256.

51 Kamili S, Sozzi V, Thompson G, Campbell K, Walker CM, Locarnini S, Krawczynski K: Efficacy of hepatitis B vaccine against antiviral drug-resistant hepatitis B virus mutants in the chimpanzee model. Hepatology 2009;49: 1483-1491.

52 Dandri M, Burda MR, Torok E, Pollok JM, Iwanska A, Sommer G, Rogiers X, Rogler CE, Gupta S, Will H, Greten H, Petersen J: Repopulation of mouse liver with human hepatocytes and in vivo infection with hepatitis B virus. Hepatology 2001;33:981-988.

53 Dandri M, Petersen J: Chimeric mouse model of hepatitis B virus infection. J Hepatol 2012; 56:493-495.

54 Lutgehetmann M, Mancke LV, Volz T, Helbig M, Allweiss L, Bornscheuer T, Pollok JM, Lohse AW, Petersen J, Urban S, Dandri M: Humanized chimeric uPA mouse model for the study of hepatitis B and D virus interactions and preclinical drug evaluation. Hepatology 2012;55:685-694.
55 Dandri M, Burda MR, Zuckerman DM, Wursthorn K, Matschl U, Pollok JM, Rogiers X, Gocht A, Köck J, Blum HE, von Weizsäcker F, Petersen J: Chronic infection with hepatitis $B$ viruses and antiviral drug evaluation in uPA mice after liver repopulation with Tupaia hepatocytes. J Hepatol 2005;42:54-60.

56 Volz T, Allweiss L, Ben MBarek M, Warlich M, Lohse AW, Pollok JM, Alexandrov A, Urban S, Petersen J, Lütgehetmann M, Dandri M: The entry inhibitor Myrcludex-B efficiently blocks intrahepatic virus spreading in humanized mice previously infected with hepatitis B virus. J Hepatol 2013;58:861-867.

57 Petersen J, Dandri M, Mier W, Lutgehetmann M, Volz T, von Weizsäcker F, Haberkorn U, Fischer L, Pollok JM, Erbes B, Seitz S, Urban $S$ : Prevention of hepatitis $B$ virus infection in vivo by entry inhibitors derived from the large envelope protein. Nat Biotechnol 2008;26: 335-341.

58 Sugiyama M, Tanaka Y, Kurbanov F, Maruyama I, Shimada T, Takahashi S, Shirai T, Hino K, Sakaida I, Mizokami M: Direct cytopathic effects of particular hepatitis $B$ virus genotypes in severe combined immunodeficiency transgenic with urokinase-type plasminogen activator mouse with human hepatocytes. Gastroenterology 2009;136:652-662. 